Leopoldo Muzzioli

\title{
Moderna concepción del Mundo Físico ${ }^{(*)}$
}

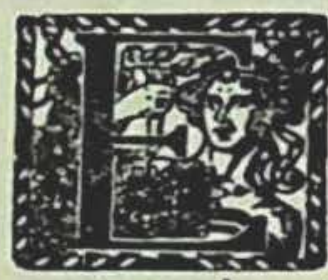

N EL AÑO 1927, es decir, hace precisamente treinta años, ganaba el concurso, por antecedentes y exámenes, de jefe de trabajos de Física en propiedad, en la Unirrera universitaria. Y creo honradamente poder afirmar que, en estos treinta años, mi mayor preocupación, mi pensamiento dominante, ha sido la Física, y he dedicado a esta hermosa rama del saber toda mi modesta actividad, con amor y entusiasmo, sea mediante la docencia o la investigación científica.

Después de todos estos años de estudio y de meditada elaboración, se ha verificado en mí lo que le sucede seguramente a todos los físicos que han vivido y viven verdaderamente esta ciencia maravillosa. Es decir, se ha realizado algo así como un extraño y trascendental proceso, por el cual la Física más que en la mente está en nuestro corazón, llega a formar parte de nosotros mismos y abarca nuestra alma.

En otros términos, no se posee esta ciencia como un simple con-

(*) Conferencia dictada el 27 de agosto de 1957, en el Salón de Honor de la Universidad, por el profesor Dr. Leopoldo Muzzioli A., Director del Instituto de Física, con ocasión del trigésimo aniversario de su carrera universitaria. 
junto de conocimientos o de noticias, sino que se tiene como traducida en un sentimiento; en un sentimiento que es de amor hacia las grandes ideas que en el desarrollo del tiempo han tratado y tratan de explicar, o, más bien, de interpretar el maravilloso misterio de la fenomenología del Universo Físico; de fervoroso agradecimiento y admiración hacia los Grandes que con su tormentoso esfuerzo han contribuido y contribuyen a su desarrollo; y también, tenemos que confesarlo, de benévolo reconocimiento por nuestra modesta contribución dada a su progreso.

A pesar que quizás habría sido mejor no mencionarlas, por ser tan íntimas y personales, èstas son las razones que me han hecho sentir como una necesidad, diría imperiosa, de organizar, en ocasión del trigésimo aniversario de mi carrera universitaria, una especie de síntesis del pensamiento científico, desde los comienzos de los estudios físicos hasta hoy día, de redactarla y expresarla.

De aquí que el tema elegido para esta disertación sea "Moderna concepción del Mundo Físico”, entendiendo por moderna concepción del mundo físico la actual interpretación dualista de los dos componentes fundamentales del Universo Físico: la energía radiante y la materia.

\section{$* * *$}

Comenzaré este estudio con la exposición del dualismo de la energía radiante y con este objeto creo oportuno exponer, antes que nada, el desarrollo en el tiempo del pensamiento científico que se refiere al fenómeno de la propagación en el espacio de la luz y de todas las otras radiaciones que son de la misma naturaleza que la luz y cuyo conjunto constituye la así llamada energía radiante. Pasaré revista a las hipótesis, las teorías, las leyes experimentales sucesivamente obtenidas y de este análisis podré hacer resaltar lo grande que ha sido y es todavía el esfuerzo y el tormento (séame permitida esta palabra) del pensamiento científico desde la iniciación de los estudios físicos hasta 
hoy día, para obtener una idea siempre más precisa, siempre más próxima a la realidad de los hechos experimentales sucesivamente descubiertos, del mecanismo (llamémoslo así) de la propagación en el espacio de la energía radiante. Y con mucha razón, los más grandes físicos del mundo se preocuparon de la propagación en el espacio de la energía radiante, por cuanto se puede afirmar que es este hecho físico tan importante el que nos da la vida.

Efectivamente, la energía radiante bajo forma de calor radiante es el gran medio de transporte de enormes cantidades de energía, y en particular es precisamente la enorme energía que nos llega del sol bajo forma de calor radiante la que nos da la vida. ¡Se trata, en efecto, de una energía verdaderamente colosal correspondiente a miles de millones de kwh! Y es una energía que no puede ser comparada, por ejemplo, con las corrientes formas mecánicas de energía, una energía de naturaleza completamente diferente; porque si la energía mecánica se manifiesta siempre acompañada por materia, la energía radiante, en cambio, parece no tener necesidad del soporte material para manifestarse; por lo tanto misterioso y complejo es el mecanismo de la propagación de esta energía y difícil será la tarea del hombre de ciencia que quiera indagar en este apasionante capítulo de la filosofía natural.

Desde la primera mitad del siglo XVII, el notable físico italiano padre Francisco María Grimaldi, aplicó el método experimental al estudio de la óptica física, como Galileo Galilei lo había aplicado a! estudio de la mecánica.

En su interesantísima y voluminosa obra Physico-mathesis de Lúmine, coloribus et irides, que consta de un Proemio y de dos Libros, y que se publicó en Bolonia en el año 1665, dos años después de su muerte, puso en evidencia, por primera vez, dos importantísimos fenómenos de óptica física: la difracción y la interferencia de la luz.

Puso en evidencia la difracción de los rayos luminosos, es decir, su desviación (en particulares condiciones) del simple comportamiento geométrico que sus predecesores habran conocido, y suficiente para explicar los más sencillos fenómenos de reflexión y refracción. Puso en 
evidencia además la interferencia, que se verifica cuando dos conos de luz provenientes de pequeños orificios cercanos, se superponen; y como consecuencia de este hecho, Grimaldi fue el primero en afirmar que la luz, superpuesta a luz, puede dar oscuridad; expresando esta verdad física con la sugestiva y audaz (en aquel tiempo) ecuación: luz más luz (en determinadas condiciones) es igual a oscuridad. Grimaldi, que con estos dos descubrimientos puede considerarse el precursor o más bien el fundador de la óptica física, se dio cuenta claramente que la difracción y la interferencia podían explicarse, en forma satisfactoria, solamente interpretando el fenómeno de la propagación de la luz, como de naturaleza ondulatoria; sin embargo, para explicar los fenómenos de la reflexión y de la refracción, ya conocidos anteriormente, consideró además necesario interpretar el fenómeno de la emisión luminosa, como de naturaleza corpuscular.

Formuló, por consiguiente, una teoría que puede sintetizarse en esta forma: el medio apto para propagar la luz, que llamó "Medio Luminífero", podría considerarse como una sustancia de peso despreciable y tal de llenar todos los espacios interestelares, todos los espacios comprendidos entre los cuerpos y entre las partículas que componen los cuerpos materiales; y la luz se debería a un doble fenómeno, emisivo y ondulatorio, consistiendo precisamente en el hecho que los cuerpos luminosos emiten un sinnúmero de partículas especiales, que moviéndose con notable velocidad en el medio luminífero, lo ponen en vibración produciendo una propagación luminosa de naturaleza corpuscular y ondulatoria a la vez.

Por lo anteriormente expuesto se desprende que la contribución de Grimaldi al estudio de la luz es verdaderamente notable, en cuanto que aparte de los importantísimos descubrimientos de la difracción y de la interferencia, hechos por los cuales puede considerarse, como hemos dicho, el creador de la óptica física, también su teoría sobre la propagación de la luz, si bien no satisfaga plenamente ya que se nota en ella una unión bastante artificiosa de las hipótesis emisiva y ondulatoria, en realidad puede considerarse una previsión maravillosa de las modernas teorías, donde ondas y corpúsculos en 
vez de contraponerse, se armonizan y son consideradas manifestaciones diferentes de un mismo fenómeno.

Más tarde el gran físico holandés Cristián Huygens concibió una hipótesis ondulatoria pura, a diferencia de la hipótesis mixta de Grimaldi, afirmando en forma explícita que las partículas de los cuerpos luminosos, encontrándose en un estado de agitación rapidísima, hacen vibrar un medio especial que él llamó "Eter" y que llenaría todos los espacios comprendidos entre los cuerpos y las partículas que constituyen los cuerpos materiales como el medio luminífero de Grimaldi, dando lugar a la propagación de la luz por ondas.

Huygens, no pudiendo anticiparse a los tiempos, ya que todavía no se habían descubierto otros fenómenos de 6́ptica física, como por ejemplo la polarización de la luz, supuso que la perturbación del éter tuviese el carácter de vibración mecánica longitudinal como se verifica en el caso del sonido y fue llevado a admitir el éter como un flúido extremadamente sutil compuesto de partículas mucho más livianas que aquéllas del aire, para explicar el hecho que los astros, los cuerpos, y las partículas que constituyen los cuerpos no deben encontrar una resistencia apreciable en sus movimientos.

En su importante estudio matemático sobre la propagación de la luz, obtuvo un resultado notable, es decir, que la velocidad de la luz considerada de naturaleza ondulatoria debía ser menor en los cuerpos refrangibles (por ejemplo en el agua) que en el vacío.

El gran físico inglés Isaac Newton se opuso siempre a la teoría ondulatoria de la luz; a decir verdad, él hallaba dificultad en poner de acuerdo la teoría ondulatoria, con el hecho de la propagación rectilínea de los rayos luminosos.

Efectivamente, se presentaba natural la objeción. Si la luz se propaga por ondas como el sonido y si el sonido se propaga también detrás de los obstáculos, ¿por qué los rayos luminosos no se comportan del mismo modo? ¿por qué no iluminan también más allá de los cuerpos opacos?

En verdad los fenómenos de difracción descubiertos por Grimaldi, evidenciaban que en ciertos casos se verifica una desviación 
al comportamiento rectilíneo y Newton conocía los experimentos de su predecesor, los repitió y continuó, pero se trataba siempre de efectos despreciables en relación a aquellos que se verifican con el sonido. Sabemos ahora que esto se explica en base al valor pequeñísimo de los largos de onda de la luz visible; estos largos de onda son todos inferiores al milésimo de milímetro y Newton, no obstante haber descubierto un fenómeno de interferencia muy interesante, los anillos coloreados que lleván su nombre, no llegó a determinar los largos de onda; la falta de estos datos obligadamente lo indujo a dudar de la teoría ondulatoria y prefirió concebir la radiación luminosa como una emisión de corpúsculos especiales, que partiendo de la fuente luminosa, bombardearían el objeto iluminado. De los cálculos matemáticos que él desarrolló con esta teoría corpuscular, dedujo que la velocidad de la luz en un medio refrangible (por ejemplo en el agua) tenía que ser mayor que en el vacío, hecho que está en contradicción con la deducción de Huygens.

Se presenta, por lo tanto, en este momento la enorme importancia de un expcrimentum crucis (experimento crucial); las determinaciones de la velocidad de la luz en el vacío y en el agua.

La posibilidad de estas determinaciones no solamente hubieran proporcionado dos datos experimentales de gran valor científico, sino que también hubieran tenido un valor filosófico-científico enorme, por cuanto hubieran dado una contribución importantísima, a fin de establecer cuál de las dos teorías se debía elegir para interpretar el hecho físico de la propagación de la energía radiante. Pero desgraciadamente, en aquel tiempo, la técnica estaba muy atrasada para dar a los físicos la posibilidad de la determinación de la velocidad de la luz en el agua, ya que para tal determinación era necesario usar distancias de laboratorio, es decir, relativamente pequeñas, y dada la gran velocidad de la luz, el tiempo extremadamente pequeño empleado para recorrer una distancia relativamente corta no era posible determinario. Se trata, efectivamente, de la medida de un tiempo del orden de un diez millonésimo de segundo para distancias de unos treinta metros. 
Solamente un siglo y medio más tarde (1850), el experimentum crucis se pudo realizar; pero ya en el año 1700 , en base a consideraciones astronómicas, Römer y Brandley aportaban la primera contribución a este problema, determinando la velocidad de la luz en el espacio vacío y los dos experimentadores, si bien partían de consideraciones y hechos astronómicos completamente diferentes, dieron aproximadamente el mismo resultado y precisamente el dato importantísimo que la velocidad de la luz en el vacío es alrededor de 300,000 km. por segundo.

De manera que en la primera mitad del siglo XVIII ya la ciencia había hecho un notable progreso con respecto a la propagación de la energía radiante.

En la segunda mitad de dicho siglo se perfeccionó mucho la construcción de los telescopios y de otros instrumentos astronómicos, de los aparatos ópticos, de los cronómetros, etc. La investigación científica podía, por lo tanto, aprovechar el gran desarrollo de los métodos experimentales, que en los albores del siglo XVIII se encontraban todavía en un estado rudimentario.

Sin embargo, por efecto de una extraña crisis de la física, se detuvo en aquel tiempo el estudio tan importante de la energía radiante.

He dicho por efecto de una extraña crisis de la física, extraña crisis ya que se verificó precisamente en aquel período en que fueron muy grandes las conquistas del genio humano, especialmente en el campo de la físico-matemática. En efecto, fue precisamente en la segunda mitad del siglo XVIII que se desarrollaron los medios de investigacián matemática y los cálculos diferencial e integłal fueron estudiados $\mathrm{y}$ aplicados en todas sus formas y las ecuaciones diferenciales se revelaron como un arma poderosa mediante la cual el hombre puede investigar y deducir las leyes que rigen los fenómenos naturales.

La armonía cada vez más perfecta entre los cálculos más complejos y los fenómenos físicos, y las observaciones astronómicas siempre más perfeccionadas, llenó al mundo de admiración y dio a los 
hombres de ciencia un optimismo exagerado y a decir verdad perjudicial para el progreso científico, ya que ellos fueron inducidos a concebir el Universo como un inmenso sistema mecánico que se podía representar en forma esquemática, mediante unas pocas y simples relaciones matemáticas; así que los físicos de ese período, basados en las concepciones mecanicista y materialista del Universo, estaban convencidos que aplicando las tres leyes de la dinámica galiliana y la ley de la atracción universal newtoniana a todas las moléculas y los átomos del Universo, se podía llegar a escribir en el papel una fórmula mediante la cual se podrían resolver los enigmas del pasado y se podría prever inexorablemente la eventualidad del futuro con toda probabilidad, aun en el campo de los hechos históricos y humanos.

Por consiguiente, en aquella época los físicos no se preocuparon más de la física del éter ni de la naturaleza física de la luz; por otra parte ¿qué preocupación podría causar este problema considerado anteriormente tan importante, si podían hacerse ilusiones de haber encuadrado todas las leyes de la naturaleza en tres ecuaciones dinámicas y en una fórmula de acción a distancia?

Pero la semilla lanzada por Grimaldi debía dar nuevos frutos.

En efecto, si bien la concepción mecanicista del Universo había llevado a los físicos a creer nuevamente en la hipótesis corpuscular de Newton, considerando la propagación de la luz como debida a corpúsculos emitidos por los cuerpos luminosos regidos por leyes mecánicas, Thomas Young consideró nuevamente los fenómenos de óptica física iniciados por Grimaldi y osó volver a la teoría ondulatoria. Me he permitido decir "osó volver" ya que después de los maravillosos éxitos del mecanicismo debido a los hombres de ciencia más autorizados de aquel entonces y que lo apoyaban incondicionalmente, el regreso a la teoría ondulatoria era considerado como un acto ignorante o por lo menos de concepción retrógrada de la naturaleza.

Pero las ideas de Young fueron aprobadas y desarrolladas en forma rigurosa y genial por el gran físico francés Agustín Fresnel, 
quien afirmó que todos los fenómenos de óptica física conocidos podían solamente encuadrarse en una teoría ondulatoria desarrollada con medios matemáticos adecuados. El fenómeno, que más que todos los otros no podían explicarse sino que con la hipótesis de la naturaleza ondulatoria de la propagación de la luz, era la interferencia.

Con su clásica y conocida experiencia de los espejos de Fresnel, pudo observar claramente la interferencia de luz monocromática (de un solo color), obteniendo una sucesión de franjas y rayas alternativamente obscuras y luminosas.

Estas investigaciones experimentales no sólo confirmaron los desarrollos matemáticos de Fresnel, sino que como aplicación de éstos, daban los valores de los largos de onda correspondientes a las diversas zonas del espectro. ¡Este resultado era verdaderamente maravilloso!

El genio humano, que ya había podido dominar con la medida las enormes distancias astronómicas, calculando con notable precisión las distancias entre los cuerpos celestes y la tierra, lograba dominar con la medida los pequeñísimos largos de onda de los diferentes colores de la luz, todos inferiores al micrón (milésimo de milímetro), con una precisión verdaderamente insospechada.

Fresnel, además, estudiando la formación de las sombras, refutó la objeción principal de Newton contra la propagación por onda, demostrando que la extremada pequeñez de las ondas luminosas, en comparación a las sonoras, explicaba el hecho que los rayos luminosos no dan vuelta detrás de los obstáculos y se propagan en forma prácticamente rectilínea.

Después de estos maravillosos éxitos, el desarrollo científico de la teoría ondulatoria de la luz, según la concepción mecánico-elástica del éter, sufrió una nueva gran modificación que se presentaba revolucionaria. Esta modificación se imponía para la interpretación de un nuevo hecho de gran importancia, ya descubierto por Malus y Brewster, la polarización de la luz. De manera que si los fenómenos de interferencia y difracción exigían una naturaleza ondulatoria 
para la propagación de la luz, que se supuso longitudinal, como aquella del sonido, los fenómenos de polarización establecían que la propagación de la luz debía ser de naturaleza ondulatoria transversal.

Después de todas estas confirmaciones experimentales, la teoría ondulatoria transversal del éter tuvo una nueva confirmación por efecto de la realización, en el año 1850, del experimentum crucis, es decir, de la determinación comparativa de la velocidad de la luz en el aire y en el agua.

Dos físicos franceses, Luis Fizeau y León Foucoult, lograron determinar la velocidad de la luz en el aire y en el agua y demostraron que la velocidad de la luz en el agua es menor que la velocidad de la luz en el aire, como lo exigía la teoría ondulatoria en contraposición a la hipótesis corpuscular.

En el año 1850 quedaba, por lo tanto, establecido que la luz se propaga con un mecanismo bien preciso y determinado, es decir, por ondas debidas a vibraciones mecánico-elásticas transversales del medio vibrante, el éter cósmico.

Pero a decir verdad, esta afirmación si bien estuviese perfectamente de acuerdo (como me parece haber dejado claramente establecido) con todos los fenómenos conocidos: reflexión, refracción, interferencia, difracción y polarización y estuviese además confirmada en forma brillante por el experimentum crucis, implicaba, sin embargo, graves consecuencias conceptuales. En efecto, solamente en un sólido elástico se pueden propagar vibraciones transversales, por consiguiente ya no se podía más concebir el éter como un flúido muy tenue y de enorme movilidad sino que nada menos que como un cuerpo sólido elástico. Además, dado el enorme valor de la velocidad de la luz, la rigidez del éter tenía que ser muy superior a aquella de cualquier cuerpo sólido conocido.

¡Qué modificación revolucionaria de la concepción del medio luminífero de los precursores de la hipótesis ondulatoria, los cuales habían dado el nombre de éter a este medio, pensando en una inmensa fluidez y una movilidad excepcional! Pero, ¿cómo podrían 
los cuerpos sólidos ordinarios moverse en el éter sin encontrar resistencia?

Los planetas, por ejemplo, han efectuado siempre y siguen efectuando sus revoluciones alrededor del sol y los satélites alrededor de los planetas, pero si encontrasen una resistencia aún pequeña al movimiento, éste se retardaría y sus órbitas se restringirían gradualmente hasta terminar con la caída sobre el centro de atracción; y las observaciones astronómicas, ya muy precisas en aquel tiempo, no revelaban el menor indicio de perturbación en este sentido.

Así que se comprende perfectamente cuán grande debían ser las inquietudes de los físicos de hace un siglo para tratar de explicar este enigma. Y el misterio que envolvía al hecho tan importante de la propagación de la energía radiante, aumentaba precisamente cuando parecían haberse definido con precisión sus características.

Se hacía necesario considerar una física del éter que no fuese de carácter mecánico; pero esta evolución debía verificarse mediante el estudio de otras categorías de fenómenos, estudio que por obra de los más grandes genios físicos del siglo XIX dio lugar a una admirable como inesperada unión en la interpretación de los fenómenos eléctricos, magnéticos y luminosos, en apariencia completamente diferentes.

El gran físico italiano Alejandro Volta, con el maravilloso descubrimiento de la corriente eléctrica, marcó una nueva etapa en el estudio de la electricidad, creando la electrodinámica. Y la corriente eléctrica conducía sucesivamente al descubrimiento de los importantes fenómenos del electromagnetismo de Ampere y de la inducción electromagnética de Faraday que dieron la posibilidad al genio de Maxwell, con una interpretación más general de estos dos hechos, de crear aquella maravillosa construcción mental desarrollada matemáticamente en forma magistral, que es la teoría electromagnética del éter cósmico.

Para tener una idea de la teoría electromagnética del éter cósmico, es indispensable, antes que nada, aclarar el concepto de campo, intuido $\mathrm{y}$ verificado experimentalmente por Faraday.

Dos masas materiales cualesquiera puestas a distancia cualquie- 
ra, se atraen. Newton determinó la ley matemática fija y exacta que guía esta atracción. Aplicando esta ley al caso de los cuerpos celestes, -se logró establecer y calcular con una precisión impresionante el movimiento de los cuerpos mismos y, en particular, se logró determinar el movimiento de los planetas y calcular y prever, con gran precisión, los eclipses de sol, de luna, etc.

Así que la ley de la atracción universal newtoniana se consideró un ideal de interpretación científica de la naturaleza.

Coulomb determinó las leyes según las cuales se atraen entre ellas las cargas eléctricas y los imanes, y estas leyes... no eran sino que aquélla de Newton. Las formulaciones matemáticas de estas leyes son en efecto absolutamente idénticas.

Sin embargo, todo esto que parece tan claro y simple es en cambio uno de los problemas más complejos de la filosofía natural.

¿Por qué se verifican estas acciones? Las leyes que las guían (que, a decir verdad, son de una simplicidad y perfección maravillasas) ciertamente no bastan para satisfacer la mente para la compresión de estos fenómenos. En efecto, se formula espontáneamente la pregunta: ¿Por qué se verifican estas acciones?

El gran físico inglés Miguel Faraday fue el hombre en cuyo espíritu se formó por primera vez la idea audaz de campo. Faraday no era un matemático, sin embargo demostró tener toda la precisión y fecundidad de conceptos de un gran cultor de las ciencias exactas. Faraday trató de adquirir conocimientos sobre la naturaleza de la atracción universal y sobre la naturaleza de las atracciones y repulsiones eléctricas y magnéticas (acciones que como es sabido se verifican también en el vacío), investigando lo que sucede en el espacio que separa los cuerpos que actúan entre ellos, y llegó a la conclusión que en el espacio interpuesto entre los cuerpos que actúan, debe existir un estado particular diferente del normal.

Lo que interesa para nuestro objeto son los hechos y las ideas relativos a los campos eléctricos y magnéticos; por lo tanto, nos limitaremos al análisis de éstos. En los fenómenos eléctricos y magnéticos, Faraday, con la intuición característica del genio y exclusiva- 
mente por vía experimental, reconoció en forma explícita y segura la importancia en dicho fenómeno del espacio aún vacío de materia interpuesto entre los cuerpos sobre los cuales se ejerce la acción.

Esta concepción lo indujo a considerar el espacio vacío, no solamente como un ente de características puramente geométricas sino que como algo mucho más sustancial, es decir, como un medio físico que puede entrar en un estado particular de modificación cuando transmite determinadas acciones. En otros términos, en un espacio vacío de materia donde se transmiten acciones eléctricas y magnéticas, se tiene un estado particular de modificación diferente del normal y este estado de modificación (deseo dejarlo bien en claro) no es una hipótesis física, sino que es un hecho real controlado por la experiencia.

Limaduras de hierro o pequeños cristales de yeso, respectivamente, en los casos del campo magnético y del campo eléctrico, pueden foner en evidencia ( $s i$ bien en forma rudimentaria, pero bastante expresiva) un campo magnético o un campo eléctrico, formando con estos dos cuerpos adecuados las líneas de fuerza del campo, el cual puede así ser representado en forma clara y explícita.

Faraday vislumbró las leyes según las cuales el estado del campo en un punto depende del estado de los puntos vecinos y las leyes de la vieja teoría de la acción a distancia debían considerarse como leyes integrales que expresaban el resultado final de todas las perturbaciones. Las leyes que Faraday vislumbró son leyes elementales, diferenciales, en comparación con las leyes globales, integrales, de Newton y Coulomb.

Así que las acciones que se verifican entre las cargas eléctricas y los imanes según la nueva concepción, no son ya más acciones a distancia, el medio interpuesto (aún vacío de materia) tiene una importancia decisiva: en él se genera un campo que transmite las acciones.

Meditando sobre la concepción de campo se puede decir, como antes to habíamos mencionado, que el espacio aún vacío de materia no puede, no debe considerarse como un ente de características sola- 
mente geométricas sino que algo mucho más substancial, algo que puede ser el soporte (séame permitida esta palabra) de campos eléctricos y magnéticos.

Por mucho tiempo, el único aspecto de los trabajos de Faraday que se tomó en consideración, fue la relación positiva de numerosas y elegantes experiencias efectuadas por él, pero el aspecto especulativo, profundo de concepción y expresado solamente con un lenguaje descriptivo y sin forma matemática, se presentaba muy difícil para ser seguido y entendido en su significado más íntimo, así que aquel aspecto de los trabajos de Faraday más importante y genial fue despreciado por sus contemporáneos y juzgado superficialmente como impreciso y no matemático. Por ejemplo, las experiencias que expresan un campo eléctrico o magnético por medio de pequeños cristales de yeso o limaduras de hierro fueron consideradas, como ahora también en muchos casos, como experiencias pueriles; en cambio, me atrevería a decir que todos, he dicho todos, los fenómenos físicos más importantes que dan lugar a la existencia y la vida del Universo residen, precisamente, en el espacio vacío; en efecto, es en el espacio vacío donde actúan y se propagan las acciones y las fuerzas que unen los componentes de los átomos, electrones superficiales y núcleos que dan lugar a la existencia y a la formación de los átomos mismos; es en el espacio vacío donde actúan y se propagan las acciones y las fuerzas que unen los átomos y que dan lugar a la formación y a la existencia de las moléculas; es en el espacio vacío donde actúan y se propagan las acciones y las fuerzas que unen las moléculas y que dan lugar a la formación de los cuerpos materiales; y la propagación misma de la energía radiante (como veremos luego) es en el espacio vacío donde se manifiesta, donde reside, donde reina: el campo es, por lo tanto, el "no materia" en un estado particular de modificación y es de enorme importancia para la vida y la existencia del Universo.

El sumo físico inglés Santiago Maxwell, en base a la concepción de campo y por medio de un análisis matemático maravilloso, dio a los fenómenos eléctricos y magnéticos y, en particular, al electromagnetismo de Ampere y a la inducción electromagnética de Faraday, 
una interpretación nueva y genial que entusiasma y sorprende al mismo tiempo.

Pocas cosas en la física logran la perfección de las ecuaciones maxwellianas, su precisión, su elegancia clásica, su profundidad. Un mundo entero se encuentra encerrado en dos líneas.

Sin embargo, dado el aspecto expresamente conceptual de esta disertación, no me es posible aclarar la teoría electromagnética de Maxwell mediante el auxilio matemático, así que creo conveniente limitarme a afirmar que Maxwell, con su teoría, logró encontrar una íntima interdeper:dencia entre los campos eléctricos y magnéticos adivinados por Faraday, cuya consecuencia le dio la posibilidad de prever la existencia, en la naturaleza de ondas electromagnéticas, que en la eventualidad de poder obtenerse, produciéndolas por medios experimentales adecuados, deberían propagarse en el espacio (aún vacío de materia) con una velocidad bien determinada y finita.

En su análisis Maxwell dedujo también la expresión matemática de la velocidad de propagación que deberían tener estas ondas electromagnéticas, y encontró que esta velocidad depende de las constantes, electroestática y magnetoestática, del medio donde se propagan, es decir, depende de la constante dieléctrica y de la permeabilidad magnética, cuyos valores, por ejemplo, en el espacio vacío, ya habían sido determinados experimentalmente. Ahora bien, reemplazando en la expresión analítica deducida estos dos datos experimentales conocidos, encontró el sorprendente resultado que la velocidad de propagación de estas eventuales ondas electromagnéticas es precisamente igual a la velocidad de la luz.

Y he aquí la grandiosa conclusión de Maxwell: la luz es un fenómeno electromagnético, las ondas luminosas no son otra cosa que un caso particular de las ondas electromagnéticas de altísima frecuencia.

Una onda luminosa está caracterizada en cada punto por dos campos; el campo eléctrico y el campo magnético que vibran, varían con la frecuencia de la onda y se propagan en la dirección de propagación. 
¿Qué transformación revolucionaria y admirable de la concepción del éter cósmico!

Hemos visto anteriormente que por efecto de los hechos experimentales conocidos: reflexión, refracción, difracción, interferencia, polarización, la luz debía considerarse de naturaleza ondulatoria y transversal; pero hemos visto también las enormes dificultades a que daba lugar esta concepción con respecto a las propiedades que debía tener el medio que debía transmitir las ondas luminosas. Este medio debía ser nada menos que de naturaleza elástico-sólida.

Se hacía necesario considerar, como dije anteriormente, una física del éter que no fuese de carácter mecánico y esta evolución debía verificarse por medio del estudio de otras categorías de fenómenos.

Esta evolución resultó efectivamente como consecuencia de la teoría electromagnética.

En la teoría electromagnética las perturbaciones alternativas no tienen más el carácter de oscilaciones mecánicas, son, en cambio, modificaciones eléctricas y magnéticas del estado neutro y la unión entre una capa y otra del medio no es más de carácter elástico mecánico, sino que substituida por la inducción mutua eléctrica y magnética.

El éter cósmico, que en su infancia con Grimaldi era un flúido extremadamente tenue, en su juventud con Fresnel era un cuerpo elástico sólido de gran rigidez; en su madurez con Maxwell es ni más ni menos que el mismo espacio vacío provisto de particulares propiedades electromagnéticas.

Sin embargo (y deseo afirmarlo en forma clara y explícita), este nuevo éter cósmico no puede, no debe considerarse el espacio vacío de materia, de características puramente geométricas, debe considerarse el espacio vacío de materia que tiene la posibilidad, la propiedad de ser el soporte de campos eléctricos y magnéticos.

El espacio vacío de materia podría considerarse "la nada" y se caería entonces en un error fundamental de interpretación, ya que la nada no podría tener en sí misma las posibilidades, las propiedades necesarias para modificarse. Después de la interpretación de Maxwell 
se ha dicho que hablar de éter cósmico no tendría ya ningún sentido; estoy tal vez de acuerdo con esta afirmación, pero deseo insistir que no se puede, no se debe substituir la denominación de éter cósmico con aquella de espacio vacío o sencillamente espacio, por cuanto con estas dos denominaciones se tendría una idea puramente geométrica del espacio; es tal vez oportuno, en cambio, hablar de "espacio físico", pues en estas dos palabras se puede verdaderamente considerar englobada toda la esencia del medio apto a transmitir la luz.

Basados en todo lo que hemos expuesto, se ve que la óptica, el estudio de la luz, se ha transformado en un corolario, en un capítulo de la electrodinámica.

Pero la teoría de las ondas electromagnéticas había sido construida por Maxwell en forma puramente analítica y también desde el punto de vista conceptual, ciertamente no ilustrada en la forma simple y expresiva como por ejemplo ha sido expuesta en esta disertación, aún más, él, que era sobre todo físico teórico, no pudo llegar a su verificación experimental.

Bien pocos de entre sus contemporáneos la entendieron, y estando acostumbrados a la vieja escuela, en general fueron inducidos a afirmar que su teoría estaba construida solamente en el papel y po. día a lo más tener un valor teórico matemático.

Se trata efectivamente de una teoría muy revolucionaria.

Afirmar que la luz no es más que una expresión de la electricidad y del magnetismo y establecer esta expresión con una teoría difícil y compleja, producía una transformación tan radical en la interpretación de los hechos, que naturalmente debía encontrar impugnadores.

Pero cada cambio de orientación científica necesita tiempo y se hacen necesarias verificaciones experimentales para convencer a los escépticos, tanto más que solamente cuando una teoŕa es confirmada por las pruebas experimentales directas, adquiere una eficiencia, una potencia de convencimiento tal como para disipar cualquiera duda y ser la fuente de nuevos progresos y fértil de frutos inesperados. 
También cuando la teoría precede a la experiencia como en este caso de genial inspiración, adquiere vida propia y se transforma en instrumento seguro de investigación solamente cuando encuentra su confirmación en pruebas experimentales bien seguras e irrefutables.

$\mathrm{Y}$ las pruebas llegaron claras, explícitas, con el descubrimiento por parte del gran físico alemán Enrique Hertz de las ondas electromagnéticas.

No viene al caso aquí exponer los dispositivos con los cuales Hertz logró producir y propagar en el espacio ondas electromagnéticas; no basta decir que el gran físico italiano Augusto Righi, en un genial y poderoso trabajo experimental, logró demostrar que las ondas electromagnéticas se comportan exactamente como las ondas luminosas. Righi, efectivamente, logró reproducir con las ondas electromagnéticas todos los fenómenos ópticos hasta entonces conocidos: reflexión, refracción, interferencia, difracción y polarización, y comprobó que las ondas electromagnéticas cstán guiadas por las mismas leyes que se verifican para las ondas luminosas. Los hombres de ciencia de aquel tiempo, que fueron reacios a creer en la teoría maxwelliana, la aceptaron plenamente después de todos los resultados obtenidos.

Las ondas electromagnéticas, previstas por Maxwell, descubiertas por Hertz, estudiadas por Righi, aplicadas por Marconi, eran de un largo de onda muy grande: un abismo las separaba de las ondas luminosas, cuyos largos de onda, como se ha dicho, son siempre inferiores al milésimo de milímetro. También fue llenado este abismo y en este campo se puede afirmar que en pocos años, con un trabajo poderoso y admirable, los físicos experimentales superaron las predicciones más optimistas, realizando experimentalmente toda la gran familia de las ondas electromagnéticas, que desde las ondas marconianas va a las hertzianas caloríficas, luminosas hasta los rayos $\mathbf{X}$ y gamma.

Por lo tanto, a fines del siglo XIX los físicos pensaron haber interpretado plenamente la energía radiante, considerándola de naturaleza ondulatoria transversal y electromagnética. 
Así que después de tantas vicisitudes, los físicos quedaron como iluminados de una apacible claridad llena de armonía.

Sin embargo, esta tranquilidad no podía continuar por mucho tiempo; porque... iporque la ciencia no se detiene!

Inexorablemente, me permito usar esta palabra, después de una conquista de valor excepcional, la investigación de la verdad física en vez de disminuir su campo de acción, parece que aumenta sus incógnitas y son necesarios ulteriores esfuerzos y ulteriores investigaciones para poder acercarse siempre más a la verdad, para acercarse al conocimiento de la esencia de la naturaleza.

Las idealizaciones, más o menos esquemáticas que construye nuestro espíritu y que constituyen el producto de nuestras meditaciones, son susceptibles de representar ciertos aspectos de las cosas, pero contienen en su esencia limitaciones, de modo que no pueden englobar toda la riqueza y la complejidad de la realidad física de la naturaleza.

Y efectivamente, el dualismo de la energía radiante, la fucha entre las hipótesis corpuscular y ondulatoria parecía eliminado en lo que se refiere a la propagación; pero ¿cuál era la esencia de la emisión y de la absorción de la energía radiante?

Hasta el final del siglo XIX, los físicos se habían ocupado, como hemos visto, en estudiar el comportamiento de la radiación cuando dicha radiación se encuentra lejos de la fuente emisora, sin embargo, a principios del siglo $\mathrm{XX}$ bien poco, y me atrevería a decir nada, se sabe de lo que sucede en el momento en que la radiación parte de la materia y en el momento en que la radiación llega sobre la materia.

Con el espectroscopio y el fotómetro, en los últimos años del siglo XIX se encontraron algunos hechos experimentales referentes al espectro continuo emitido por los cuerpos incandescentes y que pueden considerarse resumidos en dos importantes leyes experimentales, que son las leyes de Wien y de Stefan.

Se hacía necesario explicar estas leyes experimentales. Si la luz es, en base a la teoría de Maxwell, como una propagación de ondas 
electromagnéticas similares a aquellas que provienen de un oscilador de Hertz, en las fuentes luminosas deberían existir tantos pequeños osciladores similares a aquel de Hertz. Por otra parte, una carga eléctrica que vibra con movimiento oscilatorio alrededor de una posición de reposo, puede representar tal oscilador; en consecuencia, es fácil imaginar que en los átomos y moléculas de los cuerpos existan precisamente estos elementos eléctricos y que se puedan comportar de esta manera.

Así que con esta hipótesis, Rayleigh, en 1900, elaboró una teoría mediante la cual llegó a una expresión matemática de la intensidad de la radiación localizada en una determinada frecuencia, que está en completo desacuerdos con las leyes experimentales de Wien y. de Stefan.

Ulteriores perfeccionamientos introducidos sucesivamente en los razonamientos matemáticos de Rayleigh, por Wien y por otros físicos no mejoraron mayormente la situación y los físicos teóricos de ese tiempo se encontraron sometidos a una dura prueba.

Quien resolvió el problema fue Planck.

En una importantísima memoria escrita en 1902 propone una hipótesis, que se puede afirmar, que estableció una nueva era en la física.

La luz y en general la energía radiante de los átomos o de las moléculas excitadas, no es emitida en forma continua, o como se diría en término matemático, por infinitésimos arbitrarios, sino que la radiación es emitida solamente cuando en el interior del centro emisor se alcanza un valor de la energía bien determinado y finito, aunque extremadamente pequeño.

El valor de esta cantidad de energía es llamado quantum y está dado por el producto de una constante por la frecuencia de la radiación considerada.

Y esta constante se ha demostrado de una importancia trascendental; ella figura en innumerables fenómenos, hace sentir su enorme influencia a innumerables hechos físicos, por esta razón subió al rango de constante universal y en honor al gran físico que percibió 
claramente su importancia se llama constante universal de Planck. Con esta hipótesis como fundamento y con la ayuda de razonamientos estadísticos, Planck obtuvo una expresión matemática de la intensidad de la energía emitida a una cierta frecuencia y a una cierta temperatura, que está de acuerdo en forma admirable con los datos experimentales, es decir, con las leyes de Wien y de Stefan.

Se presenta ahora espontánea una pregunta: ¿Este quantum de Planck es un ente físico real o es más bien un artificio de cálculo?

Se hace necesario descubrir nuevos fenómenos, nuevos hechos en los cuales el quantum actúe con una individualidad propia, para ver los efectos que produce y para adquirir la confianza que sea verdaderamente un ente físico y no una hipótesis matemática.

El efecto fotoeléctrico es el primer fenómeno en el cual vemos actuar el quantum y observamos sus primeros efectos.

Si bombardeamos por medio de un rayo luminoso una placa metálica, esta emite cargas negativas (electrones) y se carga, en consecuencia, con electricidad positiva. Este fenómeno que se puede verificar experimentalmente de manera muy simple, es en la actualidad utilizado prácticamente en innumerables casos; con dispositivos convenientes, las células fotoeléctricas se aplican en varios campos de la técnica del siglo $\mathrm{XX}$, por ejemplo, el cine sonoro, la telefotografía, la televisión, etc.

Fue Einstein quien intuyó que en el efecto fotoeléctrico debían intervenir los quantum de Planck. Einstein trató analíticamente el fenómeno y formuló la ley que puede expresarse en la siguiente forma: En el efecto fotoeléctrico, la energía del quantum incidente sobre la materia es igual a la energía de extracción del electrón más la energía cinética adquirida por el electrón que se ha liberado de la materia.

La relación de Einstein se refiere al efecto producido por un sólo quantum, separándolo así del resto de los demás quantum irradiados por la fuente emisora.

Vemos, por lo tanto, funcionar el quantum en forma independiente de aquellos que lo acompañan, lo observamos con individuali- 
dad propia, él adquiere así una personalidad, en otros términos, se transforma en un ente físico real.

De todo lo que precede, se ha visto que el quantum puede considerarse como un gránulo de energía, pero siempre energía; sin embargo, desde el año 1922, es decir, desde cuando el notable físico norteamericano Compton puso en evidencia un efecto, que es llamado efecto Compton, el quanta puede parangonarse a un corpúsculo: el fotón.

Esto significa que el quantum deberá poseer una cierta masa y chocando contra cuerpos de sus mismas dimensiones deberá comportarse como un corpúsculo material.

Ahora bien, mediante aquel maravilloso aparato de la física del siglo XX que es la cámara de Wilson, Compton logró verificar experimentalmente y ver que efectivamente un quantum, un fotón, cuando choca con un corpúsculo, por ejemplo un electrón, lo pone en movimiento en una determinada dirección y el quantum pierde así parte de su energía y se difunde bajo formas de un quantum más pequeño. Las direcciones del quantum incidente, del quantum difundido y del electrón de rebote están ligadas por las relaciones del paralelogramo de las cantidades de movimiento.

$Y$ es verdaderamente admirable que todo se verifica con una precisión que no se podría esperar de una experiencia tan difícil de realizarse.

Y hemos llegado al estado actual de la ciencia en este apasionante estudio de la energía radiante, es decir, hemos llegado a una concepción, interpretación dualista de esta energía, considerando el conjunto de sus tres aspectos fundamentales: la emisión por el cuerpo que la emite; la propagación, en el medio donde se propaga, y la recepción, por el cuerpo que la recibe.

He tratado de exponer este tema en la forma más clara posible y mediante una síntesis, que si bien ha sido tan rápida e intencionadamente conceptual y cualitativa, espero haya dado una idea del esfuerzo del pensamiento y de la meditación de los hombres de ciencia desde la iniciación del estudio del argumento hasta nuestros 
días, para adquirir una concepción siempre más cercana a da realidad de la naturaleza de la energía radiante.

Hemos recorrido con una rápida visión las grandiosas etapas a través de las cuales el pensamiento humano ha pasado y hemos llegado al estado actual de la ciencia donde el problema, todavía lejano a su resolución, ha adquirido aspectos mucho más complejos y difíciles que al comienzo de las investigaciones.

Los tiempos de Grimaldi y de Newton están bien lejanos y todavía hemos vuelto a una concepción dualista del fenómeno luminoso en particular y de la energía radiante en general. En aquellos tiempos, de la concepción corpuscular se pasó a la concepción ondulatoria; hoy, además de las ondas, debemos admitir también los corpúsculos.

¿Cómo podemos armonizar estas dos concepciones tan contradictorias, inherentes a un mismo fenómeno?

Antes de tratar de contestar a esta trascendental pregunta, es necesario averiguar si este misterioso dualismo es simplemente una característica de la energía radiante o es más bien un principio generalísimo en la naturaleza. En otros términos, es necesario indagar el comportamiento del otro componente fundamental del mundo físico, la materia.

¿Existe este mismo dualismo; este doble comportamiento corpuscular y ondulatorio también para la materia?

Para tratar de contestar a esta pregunta, es necesario, también en este caso, remontarse a los comienzos de la ciencia de la naturaleza, donde la idea del atomismo tuvo, desde entonces, una importancia predominante.

Quien por primera vez y en forma clara y explícita expresa la idea del atomismo es Demócrito.

No existe, afirma Demócrito, más que los átomos, que son la materia, y el espacio vacío, que es la nada; todo el resto es opinión.

Los átomos, dice Demócrito, en cantidades innumerables son las partículas últimas, indestructibles e inalterables, y son la base de la naturaleza y de todos sus fenómenos. Cada átomo no tiene otras 
propiedades que su forma geométrica inalterable y su variable y eterno movimiento.

La diferencia de todas las cosas tiene su origen simplemente por la diversidad de número, magnitud y ordenación de sus átomos.

Solamente en la opinión consiste el dulce y el amargo, en la opinión consiste el calor, el frío, la luz, los colores. No existe más que los átomos, la materia, y el espacio vacío, la nada.

Todo esto afirma categóricamente Demócrito. En su representación del mundo no hay otra cosa que el aspecto geométrico y el movimiento mecánico de los átomos.

Nos encontramos, por consiguiente, frente a una de las formas más expresivas de aquel "descoloramiento del mundo" propio de la filosofía materialista y que dio por primera vez la base para concebir el desarrollo de todos los procesos naturales según una rígida y fría causalidad. Una de las rarezas de esta concepción democrítica del Universo, que es seguida en forma idéntica por Epicuro, es la oposición radical de esta manera de pensar con el mundo religioso de aquel entonces.

Para los griegos, como se sabe, cada río era un dios, en los manantiales vivían las ninfas, en las cavernas habitaban los demonios, sin embargo, todo este maravilloso mundo mitológico, que presuponía de cada fenómeno natural la obra arbitraria e imprevisible de los dioses, venía reemplazado con una imagen de la naturaleza basada sobre la más rígida y fría causalidad.

Aún más raro es el hecho que ni Demócrito ni Epicuro negaban la existencia de los dioses; los dejaban sobrevivir como seres felices e inmortales que se interesaban solamente en ellos mismos y que no tenían acción alguna sobre el devenir del mundo que se desarrollaba por su cuenta según precisas y rígidas leyes geométricas y mecánicas.

Los filósofos griegos antes mencionados y el romano Lucrecio, que en su hermoso poema De rerum natura sigue los motivos de la concepción atomista, se limitaron simplemente a enunciar una afirmación, y no sólo dejaron esta afirmación sin demostración alguna, 
sino que además no trataron de deducir de ella las posibles verdades que se hubiesen podido obtener, con el objeto de alcanzar un efectivo y fructífero provecho de su teoría; así que esta concepción atomista del mundo, a pesar de su clara y explícita exposición, no tuvo una importancia notable y debe recordarse simplemente desde el punto de vista histórico, por ser una de las primeras posiciones interesantes del pensamiento científico en este campo.

Es necesario hacer un salto enorme, de más de dos mil años, para encontrar nuevamente la concepción atomista, pero no como simple afirmación, sino más bien como teoría organizada e idónea a la consecución de resultados aptos a explicar en forma satisfactoria el comportamiento y las leyes de los fenómenos relacionados con la materia en su conjunto macroscópico y verificables experimentalmente.

La potencia vivificadora de ideas de la representación atomista y su aptitud para indicar el camino para la explicación de los fenómenos relacionados con la materia, fue reconocida y valorizada con éxito hacia 1800 por el gran físico inglés Dalton.

Sin embargo, la hipótesis que la materia esté formada simplemente por átomos libres, no es suficiente para explicar los numerosos fenómenos que se observan en el estudio de las reacciones químicas. Esta insuficiencia fue puesta en evidencia por primera vez por el gran químico italiano Avogadro, que mediante su genial hipótesis atómico-molecular logró superar las dificultades a que daba lugar la hipótesis atómica y explicar en forma clara y satisfactoria todas las leyes químicas conocidas.

La hipótesis atómico-molecular de Avogadro, completada en forma exhaustiva por otro gran químico italiano, Canizzaro, explicó en forma tan clara y evidente todas las leyes macroscópicas conocidas, que se consideró como un ejemplo de elegante perfección clásica en la interpretación de la constitución de la materia.

Y los físicos y químicos de la mitad del siglo pasado, gracias a las geniales obras de Avogadro y Canizzaro, también en la interpretación de la constitución de la materia, quedaron (como sucedió en aquel mismo período con la interpretación maxwelliana de la ener- 
gía radiante) como iluminados de una apacible claridad llena de armonía.

Sin embargo, las trascendentales, y se podría decir revolucionarias modificaciones para la interpretación de la energía radiante, se verificaron también, casi paralelamente, para la interpretación de la materia.

Para el objetivo de esta disertación no es estrictamente necesario exponer todos los descubrimientos que se realizaron en este siglo en el campo de la Física Atómica; baste señalar que (como es bien conocido) el átomo fue estudiado y se está estudiando, teórica y experimentalmente, en forma sobre manera amplia y exhaustiva, encontrándose que está constituido por numerosos corpúsculos o entes, como el núcleo (de naturaleza extremadamente compleja) y los electrones superficiales.

Se encontraron así experimentalmente, sea directamente que por efecto de geniales previsiones teóricas, los electrones, los protones, los neutrones, los positrones, los mesones, los antiprotones, los neutrinos, etc.

Pero ahora debemos preguntarnos: y aquí está la esencia de la cuestión que nos interesa: ¿todas estas partículas elementales de hoy, cargadas o no de electricidad, debemos considerarlas en el sentido democrítico o más bien se trata de entes de naturaleza completamente diferente?

En otros términos: ¿la Física Atómica en el estado actual de la ciencia, es una confirmación, o precisión, de la concepción democrítica y daltoniana, o más bien una radical confutación de esta representación que Avogadro y Canizzaro completaron en forma magistral que se consideró tan satisfactoria?

Para tratar de contestar a esta pregunta, de verdaderamente trascendental importancia, es necesario volver nuevamente a las ideas y hechos que nos llevaron a la concepción dualista de la energía radiante.

En aquel apasionante estudio, habíamos llegado a un punto donde estábamos obligados a afirmar que un gran número de fenómenos 
naturales, verificados experimentalmente, sobre los cuales no podemos, no debemos tener ninguna duda, pueden ser interpretados solamente invocando una naturaleza corpuscular de la energía radiante; pero, por otra parte, vimos también que existe un gran número de fenómenos naturales verificados experimentalmente, sobre los cuales no podemos ni debemos tener ninguna duda, que pueden ser interpretados solamente mediante una naturaleza ondulatoria de la energía radiante; y nos preguntamos ¿cómo es posible armonizar estas dos concepciones tan contradictorias inherentes a un mismo fenómeno?

Ahora bien, dos grandes físicos: el austríaco Schrödinger y el francés De Broglie, para tratar de explicar o, mejor dicho, para tratar de obtener una interpretación satisfactoria a la concepción dualista de la energía radiante, elaboraron alrededor del año 1925 una genial teoría, la mecánica ondulatoria, que dio una contribución admirable en este sentido.

Sin embargo, la trascendencia de la mecánica ondulatoria de Schrödinger-De Broglie, desarrollada teóricamente con una agudeza, precisión y profundidad maravillosas, no se limita a esto, sino que en su interpretación física da lugar a este inesperado resultado: ¡la asombrosa previsión que el dualismo que se verifica para la energía radiante debe verificarse también para la materia!

¡También la materia tiene que manifestarse a veces como onda y a veces como corpúsculo!

Esta extrapolación, tan bella como audaz, obtuvo en seguida una confirmación experimental con el descubrimiento de la difracción de los electrones; y aquí los experimentos de Davisson, Kunsmann, Thomson, Germer, Rupp y Kikuchi, hechos entre los años 1925 y 1940, han demostrado en forma inequívoca la realidad de la previsión de la mecánica ondulatoria; así que el electrón, que hasta entonces había sido considerado como un elemento de naturaleza puramente corpuscular y cuyos atributos eran solamente su masa, su carga y su energía cinética, debe ahora considerarse de naturaleza también ondulatoria y con un largo de onda asociado de un valor bien 
determinado y preciso dependiente de su masa, de su velocidad y de la constante universal de Planck.

Por lo tanto, no solamente la energía radiante se manifiesta a veces como onda y a veces como corpúsculo, sino que también la materia tiene la posibilidad de manifestarse bajo este doble aspecto. Parece, por lo tanto, que se puede afirmar que este dualismo no es debido a una interpretación humana de los hectos sino que a un principio general ínsito en la naturaleza misma.

Nadie podía prever los resultados universales y de importancia trascendental logrados.

Las ondas electromagnéticas, la luz invisible, la luz visible, los rayos $\mathrm{X}$, los rayos gamma, las emisiones de las substancias radioactivas, los electrones, los átomos, los componentes de los átomos, vienen a tener propiedades comunes y vienen a estar unificados de un modo que nosotros todavía no logramos comprender perfectamente.

Tal vez jamás como en este momento se ha sentido y se siente 1. limitación de nuestra intuición, para comprender, para interpretar fa esencia de estos dos dualismos, sea de la energía radiante que de la materia, que los fenómenos experimentales y las expresiones analíticas dan por ciertos y verdaderos.

He dicho que, en la física clásica, el estudio de la energía radiante y de la materia (es decir, de los dos componentes fundamentales del Mundo Físico) había dado lugar a una apacible claridad llena de armonía; ahora, la luz emitida por los extraordinarios hechos e ideas de la Física Moderna produce, en cambio, un deslumbramiento casi doloroso, que perturba, emociona y a veces espanta por sus aspectos incomprensibles; no obstante es necesario, indispensable, mantener los ojos abiertos y sufrir el tormento de este deslumbramiento, porque solamente de esta manera podremos acostumbrar nuestra mente a ver e interpretar esta nueva maravillosa concepción del Universo Físico y seguir con férrea voluntad y noble esfuerzo el asombroso adelanto de la ciencia actual. 